\section{L'Actualité économique}

L'ACTUALITÉ

ÉCONOMIQUE

\section{Les disparités de salaire entre les secteurs public et privé québécois}

\section{Robert Lacroix}

Volume 59, numéro 2, juin 1983

URI : https://id.erudit.org/iderudit/601211ar

DOI : https://doi.org/10.7202/601211ar

Aller au sommaire du numéro

Éditeur(s)

HEC Montréal

ISSN

0001-771X (imprimé)

1710-3991 (numérique)

Découvrir la revue

Citer cet article

Lacroix, R. (1983). Les disparités de salaire entre les secteurs public et privé québécois. L'Actualité économique, 59(2), 181-189.

https://doi.org/10.7202/601211ar
Ce document est protégé par la loi sur le droit d'auteur. L'utilisation des services d'Érudit (y compris la reproduction) est assujettie à sa politique d'utilisation que vous pouvez consulter en ligne.

https://apropos.erudit.org/fr/usagers/politique-dutilisation/ 
L'Actualité Économique. Revue d'analyse économique, vol. 59, no 2, juin 1983.

\title{
LES DISPARITÉS DE SALAIRE ENTRE LES SECTEURS PUBLIC ET PRIVÉ QUÉBÉCOIS*
}

\author{
Robert LACROIX \\ Université de Montréal
}

On semble penser de plus en plus que les syndicats du secteur public sont tellement puissants qu'ils peuvent dicter leurs conditions de travail aux divers gouvernements. L'augmentation considérable de l'emploi public $^{1}$ se combinant à un changement graduel du salaire relatif public/privé ne sont pas étrangers au développement de cette impression. Par ailleurs, les données du Bureau de recherche sur la rémunération du Conseil du Trésor du Québec indiquant qu'en 1979, pour des emplois comparables, la rémunération globale était de quelque $16 \%$ plus élevée dans le secteur public confirmaient une poussée de la rémunération dans ce secteur ${ }^{2}$.

Est-ce que l'on vit actuellement les conséquences d'un pouvoir syndical dont on ne connaît pas encore les limites? On ne peut répondre à cette question sans analyser sommairement le processus de détermination des salaires dans le secteur public et voir si le comportement des agents économiques dans ce secteur et les conditions économiques qui les caractérisent sont susceptibles de conduire à des niveaux et des évolutions de salaire différents dans les secteurs public et privé.

\section{La détermination des salaires dans le secteur public}

On s'entend généralement pour dire que l'objectif syndical n'est pas différent dans le secteur public de ce qu'il est dans le secteur privé. Le syndicat cherche à obtenir l'entente salariale (ou les conditions de travail) la plus avantageuse possible pour ses membres. Les crières minimum

*Allocution du président de la Société canadienne de science économique prononcée lors du Congrès annuel de mai 1982. Je remercie mes collègues J.M. Cousineau, A. Hollander, R. Houle, M. Gaudry, C. Montmarquette, Y. Rabeau, A. Raynauld et F. Vaillancourt pour leurs nombreux commentaires sur une première version de ce texte.

1. Foot and Thadaney (1978).

2. Bureau de recherche sur la rémunération (1979): chaque fois que nous parlerons du secteur public, nous entendrons les secteurs public et parapublic. Une nouvelle étude du Conseil du Trésor montrerait que cet écart est maintenant de $11 \%$. Cet écart est une moyenne et il y a une variation de l'écart selon les catégories d'emploi. 
d'évaluation de la qualité d'une entente salariale par les syndiqués sont les conditions que des travailleurs comparables ont pu obtenir dans d'autres négociations et l'incidence de l'entente salariale sur leurs emplois. Les dirigeants syndicaux qui n'obtiennent pas d'ententes salariales satisfaisantes selon ces critères sont menacés dans leur leadership. Indépendamment du secteur d'activité, les dirigeants syndicaux sont donc incités à obtenir les ententes salariales les plus avantageuses possibles compte tenu des contraintes d'emploi.

C'est surtout au niveau de l'employeur que la situation se différencie entre les secteurs public et privé. Dans le secteur privé, l'objectif visé par l'employeur lors d'une négociation salariale est d'abord et avant tout le maintien de coûts unitaires comparables à ceux de ses concurrents sur le marché des produits. Une «mauvaise» entente salariale signée par l'entreprise entraînera une sanction presque immédiate. En effet, il devra y avoir une augmentation relative du prix de ses produits (ou une baisse du taux de profit) et une perte subséquente d'une partie de son marché. L'entreprise a donc intérêt, elle aussi, à rechercher la meilleure entente salariale possible. Les critères d'évaluation d'une bonne entente salariale pour une entreprise sont le maintien et de sa position concurrentielle en terme de coûts et de sa capacité d'attirer, aux salaires de l'entente, les travailleurs dont elle a besoin. On réalise que la contrainte de l'entreprise dépendra de son pouvoir sur le marché des produits.

On est porté à inférer de ce modèle de comportement des agents économiques du secteur privé les conséquences de la position particulière du secteur public sur le marché des produits. En effet, les gouvernements sont en position de monopole pour un grand nombre de services qu'ils offrent. Qui plus est, ces services publics qui ont un coût n'ont souvent pas de prix de marché. Ils ne sont pas vendus mais distribués à la population. De ce fait, on affirme régulièrement que les gouvernements ne subissent pas la sanction du marché et ont, par conséquent, moins d'incitation à résister aux demandes syndicales, d'où le biais inflationniste dans les ententes salariales du secteur public.

Cette conclusion doit être nuancée si l'on se fie aux travaux des auteurs qui ont récemment tenté d'élaborer une théorie de l'emploi et des salaires dans le secteur public ${ }^{3}$. En effet, les décisions salariales des gouvernements ne sont pas sanctionnés sur le marché des produits, mais bien sur le marché politique. Et ce marché impose aussi des contraintes et des sanctions aux employeurs du secteur public. Si l'on accepte que l'objectif premier d'un homme politique est de garder le pouvoir et donc, en

3. Sans être exhaustif, soulignons les auteurs suivants: Fogel et Levine (1974); Reder (1975); Ehrenberg et Goldstein (1975); Cousineau et Lacroix (1977); Dussault (1978). 
démocratie, d'être réélu, on doit se demander en quoi l'atteinte de cet objectif pourrait être partiellement ou totalement compromis par les ententes salariales du secteur public. Partant d'une situation d'équilibre, dans la mesure où une entente salariale du secteur public affectera la taxation ou l'endettement de l'État, elle nuira à l'atteinte de l'objectif premier de l'homme politique. En ce sens, toutes choses étant égales par ailleurs, une entente salariale sera jugée bonne par l'employeur public si elle permet de maintenir la quantité et la qualité des services existants en conservant à son emploi une main-d'œuvre de qualité requise sans augmentation de taxe ou de déficit. Les hommes politiques ont donc tout intérêt à ce que de «bonnes» ententes salariales soient signées dans le secteur public puisque s'il en est autrement ou bien les taxes (ou le déficit) augmenteront ou bien la qualité des services offerts diminuera et il s'ensuivra une perte de votes et éventuellement la perte du pouvoir. Il est donc évident qu'autant dans le secteur public que dans le secteur privé, les ententes salariales résultent de décisions sous contraintes.

A savoir si ces contraintes sont plus restrictives à long terme dans l'un ou l'autre des secteurs devient une question empirique à laquelle on peut partiellement répondre en évaluant l'élasticité de la demande de travail par rapport aux salaires dans les deux secteurs. Quelques auteurs américains ont tenté d'effectuer des estimations de ces élasticités. Il ressort de ces études qu'aux États-Unis l'élasticité de la demande de travail par rapport aux salaires se concentrerait aux alentour de $-0,5$ à $-1,0$ dans le secteur privé et aux alentours de $-0,5$ dans le secteur public. L'écart du simple au double serait pour ces auteurs un grand maximum ${ }^{4}$. Partant de cet écart maximum, supposons que les salaires minimums de recrutement d'un secteur public non syndiqué sont les salaires du secteur privé non syndiqué et que l'écart salarial entre les syndiqués et les non syndiqués du secteur privé est de quelque $7 \%$. Si les objectifs d'emploi des syndicats du secteur public ne sont pas différents de ceux du secteur privé, la syndicalisation des travailleurs du secteur public pourra conduire à terme à un écart maximum de salaire entre les syndiqués du secteur public et ceux du secteur privé, favorable aux travailleurs du secteur public et s'élevant à quelque $7 \%$.

Une syndicalisation complète du secteur public telle qu'elle existe au Québec ne donne-t-elle pas aux syndicats, à cause du caractère essentiel des services publics, un pouvoir tel qu'ils pourront et maintenir leur emploi et creuser encore davantage l'écart entre les salaires versés dans le secteur public et ceux du secteur privé? En d'autres mots, par les coûts

4. Voir entre autres Ashenfelter et Ehrenberg (1975).

5. C'est le genre de résultats auxquels on arrive dans Cousineau, J.-M., R. Lacroix et F. Vaillancourt (1982). 
qu'une grève générale imposerait à la population, les syndicats ne la forceront-elle pas à accepter des écarts de salaire plus considérables entre les deux secteurs et à donner au gouvernement les signaux correspondants sur le marché politique?

Dissipons d'abord une certaine confusion qui semble exister entre le caractère essentiel des services publics et les conséquences de la centralisation des négociations avec regroupement en un front commun des forces syndicales tel qu'on le trouve au Québec. Si on avait la même centralisation des négociations dans le secteur privé qu'on a dans le secteur public, on pourrait, par exemple, aboutir à ce que l'ensemble de l'industrie des aliments et boissons soit en grève. Les coûts d'une telle grève seraient probablement aussi élevés sinon plus que ceux d'une grève dans le secteur de l'éducation. On réalise donc que peu importe le secteur, la centralisation des négociations augmente toujours le coût que les parties non impliquées directement dans la grève doivent supporter. Je suis donc porté à penser que concernant les négociations collectives, le secteur public québécois se différencie du secteur privé davantage par la centralisation des négociations que par le caractère essentiel des services qu'il offre.

Ceci dit, est-ce que le caractère essentiel de certains services publics et surtout la centralisation des négociations ne donnent pas aux syndicats du secteur public du Québec un pouvoir particulier?

Comme nous l'avons souligné antérieurement, la dimension des coûts d'une grève généralisée dans les secteurs public et parapublic est tellement considérable que la population ne peut pas les ignorer. En conséquence, elle appuiera, jusqu'à une certaine limite le gouvernement qui tentera de les éviter par des ententes salariales plus généreuses. Les études de Rabeau (1981) sur la détermination des salaires dans les secteurs de l'éducation et de la santé au Canada et sur l'incidence de la centralisation des négociations sur les ententes salariales, appuient nettement l'hypothèse précitée. Par ailleurs, les comparaisons de salaire pour un certain nombre d'emplois repères des secteurs public et parapublic du Québec et de l'Ontario vont dans le même sens. En effet, alors que dans le secteur privé les salaires au Québec sont, à toutes fins pratiques, à parité avec ceux de l'Ontatio ${ }^{6}$, dans les secteurs public et parapublic, les salaires seraient de façon générale nettement plus élevés au Québec ${ }^{7}$. Quoique les écarts

6. Cousineau, Lacroix, Vaillancourt (1982), op. cit.

7. Bureau de la recherche sur la rémunération, Comparaison de la rémunération de certains emplois repères principaux de la fonction publique du Québec et du gouvernement de l'Ontario. Conseil du Trésor, Québec, janvier 1981 ; Comparaison entre la rémunération des enseignants au Québec et dans les principales provinces canadiennes, Conseil du Trésor, Québec, décembre 1980. 
soient variables d'un type d'emplois à l'autre (allant jusqu'à $30 \%$ dans certains cas) je pense être conservateur en disant qu'en moyenne l'écart entre les salaires des secteurs public et parapublic du Québec et de l'Ontario se situe aux alentours de $10 \%$. Il semble donc que le potentiel d'écart entre les salaires du secteur privé et ceux du secteur public du Québec est particulièrement élevé par rapport à la norme canadienne et probablement nord-américaine.

Nous avons dit antérieurement que face aux coûts anticipés d'une grève généralisée dans les secteurs public et parapublic, le gouvernement pouvait céder, avec un certain accord des voteurs, des ententes salariales plus généreuses qu'autrement. Il y a évidemment une limite à payer pour éviter des coûts. Si les syndicats abusent de la grève pour obtenir des conditions de travail de plus en plus avantageuses, la résistance des employeurs publics, malgré les inconvénients de la grève, peut devenir rentable politiquement et la capacité des syndicats d'obtenir des concessions sous la menace de la grève sera grandement atténuée. Le renversement de la situation peut même être tel que la majorité des voteurs appuieraient un gouvernement qui remettrait en cause le droit de grève dans le secteur public. En ce sens, personne ne contestera qu'il y a une limite au pouvoir syndical dans le secteur public. Reste à savoir si elle n'est pas si éloignée qu'avant de l'atteindre, des torts considérables auront été faits à l'économie.

\section{Les conséquences des disparités de salaire entre les secteurs privé et public}

Les disparités de salaire entre les secteurs public et privé nuiront à la croissance et à la stabilité de l'économie si, entre autres choses, les ententes salariales du secteur public ont des effets d'entraînement sur celles du secteur privé. Dans une étude récente que F. Dussault et moi-même avons faite, nous avons démontré qu'on devait s'attendre d'un point de vue théorique à l'existence de tels effets d'entraînement et nous les avons estimés empiriquement ${ }^{8}$. La conclusion principale de cette étude est que l'évolution des salaires du secteur public affecte de façon significative celle des salaires du secteur privé.

A partir de là, tentons de voir les conséquences possibles de l'établissement et même de l'élargissement, pendant un temps, de disparités de salaire (ou de rémunération globale) entre les secteurs public et privé. Dans un premier temps, dû aux effets d'entraînement, il y aura des pressions à la hausse sur les salaires du secteur privé. Ces pressions auront d'autant plus de chance de se transformer en augmentations de salaire que les employeurs qui les subissent ne sont pas exposés à la concurrence nationale et internationale. Dans le secteur exposé de l'économie, l'effet

8. Dussault, F. et R. Lacroix (1980). 
d'entraînement du secteur public sera plus faible à cause de la plus grande résistance des employeurs mais l'activité de grève s'y accroîtra fortement. L'effet d'entraînement sera plus considérable dans le secteur abrité puisqu'une partie de l'ajustement pourra être refilée en prix. Ces ajustements salariaux entraîneront des modifications de l'emploi et du chômage. A cause de la relative insensibilité à la baisse des salaires aux conditions d'offre excédentaire sur le marché du travail, le chômage supplémentaire persistera si rien n'est modifié par ailleurs. Il faut, toutefois, remarquer que, dû aux effets d'entraînement, les disparités initiales de salaire entre les secteurs privé et public ont été réduites. Advenant que les syndicats du secteur public veuillent rétablir l'écart initial ou davantage, les mêmes effets se reproduiront. Or, si la politique des syndicats du secteur public est de faire de ce secteur la «locomotive» du train salarial de l'économie, c'est ce qui arrivera. Apparaîtra alors un problème de croissance de l'emploi à cause de la diminution de la capacité concurrentielle des entreprises et le chômage augmentera.

Dans ces conditions, le gouvernement se sentira obligé de remédier à la situation en bonifiant les programmes d'assistance sociale et en stimulant lui-même la création d'emploi par des projets d'investissements publics, d'emploi communautaire, de rachats d'entreprises à la veille de fermer leur porte, etc. Pour ce faire, ce même gouvernement sera amené à augmenter les taxes aux individus et aux entreprises. Ces augmentations de taxes diminueront davantage la capacité concurrentielle des entreprises et hausseront les réclamations salariales de tous les travailleurs y compris ceux du secteur public. Le climat général des relations de travail se détériorera encore plus, la croissance économique diminuera et la situation financière de l'État sera de plus en plus précaire. On aura probablement atteint la limite dont je parlais antérieurement, mais les torts causés à l'économie auront été considérables.

Je pense que ce scénario n'est pas tellement loin de ce que le Québec a vécu au cours de la dernière décennie. Cette affirmation n'est pas gratuite mais s'appuie sur les résultats d'une étude récente que Cousineau, Vaillancourt et moi-même avons faite sur l'évolution des marchés du travail de Montréal métropolitain et de Toronto métropolitain de 1970 à $1980^{9}$. Cette étude montre comment une poussée surtout institutionnelle des salaires à Montréal au cours de ces dix années a entrainé d'abord une réduction des écarts salariaux entre Montréal et Toronto pour ensuite les renverser. Cette poussée salariale s'est accompagnée d'une activité de grève deux fois plus élevées à Montréal qu'à Toronto, d'une baisse considérable de l'emploi relatif et de la population relative.

9. Cousineau, Lacroix et Vaillancourt (1982), op. cit. 
Au total, il semble que les contraintes imposées par le marché politique sur la détermination des salaires dans le secteur public du Québec n'ont pas été suffisantes pour éviter une glissement dommageable des salaires versés aux employés de l'État.

\section{Quelles sont les solutions?}

Un premier élément de solution serait un renforcement du rôle du marché politique dans la détermination des salaires dans le secteur public. Je vois deux façons complémentaires de le faire.

D'abord, le maximum d'informations devraient régulièrement être fournies à la population, par un organisme indépendant, sur les comparaisons de salaire dans les secteurs public et privé, dans le secteur public du Québec et ceux des autres provinces et en particulier l'Ontario, sur l'état de l'offre excédentaire pour les emplois du secteur public, sur les taux de roulement de la main-d'ouvre dans ce secteur... et sur l'incidence de l'évolution des salaires du secteur public sur les finances publiques. On éviterait ainsi, d'une part, que les hommes politiques ne fournissent des informations que lorsqu'elles servent leurs fins et, d'autre part, de réaliser ex post seulement, qu'un sérieux dérapage s'est produit dans les conditions de travail consenties aux travailleurs du secteur public. Une population bien informée sur une question limite considérablement la marge de manœuvre des gouvernements.

Ensuite, le gouvernement doit pouvoir le moins possible dépenser sans taxer «explicitement». On sait qu'en l'absence d'indexation des impôts, l'inflation gonfle les recettes de l'État de façon automatique. Il devient alors possible pour un gouvernement de satisfaire des demandes salariales élevées sans augmentation de la taxation «explicite» ni du déficit. Le coût politique de l'augmentation salariale est faible, si l'on se réfère aux payeurs de taxes, et l'avantage peut être grand, si l'on tient compte des gains de votes auprès des travailleurs du secteur public. Dans le cas du Québec, les salaires des secteurs public et parapublic comptant pour plus de $50 \%$ du budget, une indexation automatique des impôts sur le revenu comme elle existe dans le reste du Canada, aurait probablement freiné la croissance des salaires dans le secteur public au cours de la dernière décennie. Personne, je pense, ne s'opposerait à ce que dans le domaine de l'indexation des impôts, le Québec devienne une province comme les autres. Cette deuxième mesure réduirait, elle aussi, la marge de manœuvre du gouvernement dans les négociations salariales.

A côté de ces mesures générales, le gouvernement devrait clairement préciser sa philosophie salariale sur les deux points suivants. Premièrement, comme il serait pratiquement suicidaire pour l'économie du Québec de faire du secteur public le secteur leader dans les ententes salariales, 
le gouvernement doit s'opposer fermement à la volonté syndicale de faire des salaires dans le secteur public la «locomotive» du train salarial de l'économie du Québec. Deuxièmement, les salaires dans le secteur public ne doivent pas être utilisés par le gouvernement pour corriger une distribution des revenus qui lui paraitrait incorrecte. D'une part, c'est probablement la façon la moins efficace du point de vue économique de redistribuer le revenu et, d'autre part, je ne vois pas pourquoi un groupe particulier d'individus, soit les travailleurs du secteur public, seraient privilégiés.

Enfin, des mesures particulières devraient être prises pour que soient ' considérablement réduits les coûts que les parties impliquées dans un conflit de travail du secteur public font supporter à la population. Comme il serait irréaliste de préconiser un retour à des négociations complètement décentralisées ou un retrait pur et simple du droit de grève, on ne peut que souhaiter deux choses. D'abord, que le gouvernement évite toute mesure qui accroîtrait encore davantage la centralisation des négociations. Ensuite, que soient déterminés par le gouvernement les services essentiels qui devraient être assurés lors de tout conflit de travail dans les différents services publics.

\section{BIBLIOGRAPHIE}

Annable, James E. (1974), "A Theory of Wage Determination in Public Employment", Quarterly Review of Economics and Business, hiver 1974, pp. 44-58.

Ashenfelter, Orley C. et R.G. Ehrenberg (1975), «The Demand for Labor in the Public Sector», in Labor in the Public and Non-profit Sectors, Hammermesh, D.S. (éd.), Princeton University Press.

BuREAU DE RECHERCHE SUR LA RÉmUNÉRATION (1979), Comparaison de la rémunération entre le secteur public et parapublic et le secteur privé au Québec, Québec, Conseil du Trésor.

BUREAU DE RECHERCHE SUR LA RÉMUNÉRATION (1980), Comparaison entre la rémunération des enseignants au Québec et dans les principales provinces canadiennes, Conseil du Trésor, Québec. 
Bureau de RECherChe SUR LA RÉmunéRATION (1981) Comparaison de la rémunération de certains emplois repérés principaux de la fonction publique du Québec et du gouvernement de l'Ontario, Conseil du Trésor, Québec.

Conseil ÉCONOMiQue du CANAda (1974), L'énigme de l'inflation, Treizième exposé annuel, Ottawa.

Cousineau, JEAN-MiChel et R. LACROIX (1977), La détermination des salaires dans le monde des grandes conventions collectives: Une analyse des secteurs privé et public, Conseil économique du Canada, Ottawa.

Cousineau, Jean-Michel, R. Lacroix et F. Vaillancourt (1982), Les marchés du travail de Montréal et de Toronto, CETAI (HEC).

DusSAult, FrançoIS (1978), La syndicalisation du secteur public municipal et la détermination des salaires, Mémoire de maîtrise, département des sciences économiques, Université de Montréal.

Dussault, François et R. Lacroix (1980), "The Spillover Effect of Publicsector Wage Contracts in Canada», Cahier 8105, Département des sciences économiques, Université de Montréal.

Ehrenberg, Ronald G. et G.S. Goldstein (1975), «A Model of Public Sector Wage Determination", Journal of Urban Economics, pp. 223-245.

Fogel, Walter et D. Lewin (1974), "Wage Determination in the Public Sector", Industrial and Labor Relations Review, 27, pp. 410-431.

Foot, David D. et P. Thadaney (1978), "The Growth of Public Employment in Canada: The Evidence from Taxation Statistics 1946-75", in Public Employment and Compensation in Canada: Mythe and Realities, D. Foot (éd.), IRP, Montréal 1978.

Reder, Melvin W. (1975), "The Theory of Employment and Wages in the Public Sector», dans Hammermesh, D.S. (éd.) (1975). 